\title{
KATALOG DER GRIECHISCHEN HANDSCHRIFTEN IM BESITZ DER THÜRINCER UNIVERSITÄTS- UND LANDESBIBLIOTHEK JENA $^{1}$
}

\author{
ANNETTE VON STOCKHAUSEN/ERLANCEN
}

Mlit 8 Abb. auf Tafel XIII-XX

\section{Geschichte der Sammlung2}

Im Besitz der Thüringer Universitäts- und Landesbibliothek Jena befindet sich eine kleine Sammlung griechischer Handschriften. Sie umfaßt heute fünf Codices, die aus Nachlässen verschiedener Jenaer Gelehrter zwischen dem 17. und dem 19. Jahrhundert an die Bibliothek gekommen sind.

Unter dein Nachlaß des Geschichtsprofessors Johann Andreas Bose ${ }^{3}$ (1626-1674), der von der Universitätsbibliothek 1676 erworben wurde, befanden sich zwei griechische Handschriften, die heute die Signaturen Ms. Bos. f. 1 und Ms. Bos. q. 7 tragen. Aus dem Besitz des Theologen Johann Jakob Griesbach ${ }^{4}$ (1745-1812) stammt eine Handschrift (Ms. prov. o. 25), die mit dem größeren Teil der Bibliothek Griesbachs Ende 1815 an die Universitätsbibliothek gelangte. Eine Handschrift, Ms. G.B. q. $6^{\mathrm{a}}$, wurde der Universitätsbibliothek von Wolfgang Maximilian Frhr. von Goethe ${ }^{\bar{j}}$ (1820-1883), dem Enkel Wolfgang von Goethes, zusammen mit seinen übrigen Bü-

${ }^{1}$ Herrn Prof. Schreiner (Köln) gilt mein Dank für die.vielfältige Unterstützung und Hilfe. Herrn Prof. Harlfinger (Hamburg) für die Identifizierung einiger der Schreibcr. Für wichtige Hinweise zu Ms. G. B. f. 6a danke ich Frau Prof. Velkovska (Rom). Die Abbildungen erfolgen mit freundlicher Genehmigung der Thüringer Universitäts- und Landesbibliothek Jena, deren Abteilung Handschriften und Sondersanmlungen jederzeit großes Entgegcnkommen zeigte.

2 . I'gl. Handschriften und Alte Drucke aus den Sammlıngen der Universitätsbibliothek der Fricdrich-Schiller-Universität Jena, ed. G. Karpe unter Mitwirkung von I. Kratzsch und H. Vogt, Jena o.J. Zur Geschichte der Universitätsbibliothek allgemein vgl. Geschichte der Universitätsbibliothek Jena 1549-1945, bearbeitet von einer Arbeitsgemeinschaft wissenschaftlicher Bibliothekare der Universitätsbibliothek Jena. Im Namen der Mitarbeiter K. Bulling, Weimar 1958.

3 Vgl. C. G. Jöcher, Allgemeines Gelehrten-Lexicon 1, Leipzig 1750, 1270; H. Kappner, Die Geschichtswissenschaft an der Universität Jena vom Humanismus bis zur Aufklärung, Zeitschrift des Vereins für Thüringische Geschichte und Altertumskunde N.F. 14 ff., Beiträge zur Geschichte der Universität Jena H. 3, Jena 1931, 68-131; J. Günther, Lebensskizzen der Professoren der Universität Jena seit 1558 bis 1858: Eine Festgabe zur 300jährigen Säcularfeier der Universität am 15., 16. und 17. August 1858, Jena 1858, 182.

+ Allgemeine Deutsche Biographie 9, 1879, 660-663; Günther, Lebensskizzeny (wie Anm. 3), 26; G. Delling, Johann Jakob Griesbach. Seine Zeit, sein Leben, sein Werk, Theologische Zeitschrift 33 (1977) 81-99; B. M. Metzger, Art. Griesbach, Theologische Realenzyklopädie 14, 1985, 253-256.

5 B. Gajek, Art. Coethe 5) Maximilian Wolfgang Frhr. v., Neue Deutsche Biographie 6 (1964) 5766f. Er verfaßte u. a. auch "Studien und Forschungen über das Leben und die Zeit des Cardinals Bessarion 1395-1472: Abhandlungen, Regesten und Collectaneen "(Jena 1871). 
chern im Jahr 1880 vermacht $^{6}$. Eine weitere Handschrift, Ms. G.B. f. 31, trägt zwar ebenfalls die Signatur.der Goethe-Bibliothek ${ }^{7}$, gelangte aber aus dem Nachlaß des Medizinhistorikers Christian Gottfried Gruner ${ }^{8}$ (1744-1815) an die Universitätsbibliothek 9 .

\section{Katalog ${ }^{10}$}

\section{Bos. F. 1}

Epiphanius von Salamis / Zitate aus (Ps.-)Johannes Chrysostomus / Nicephorus von Konstantinopel

(ff. 1-120") 20.3.1304 / (ff. 121-168") um 1300 / (ff. 169-174) 2. Viertel 14. Jh. Bombyzin I, 174, I' $322 \times 226 \mathrm{~mm}$ (Spiegel) Alte Signatur „Fol. 174“. Marginalnotizen von vier verschiedenen Händen.

1 (ff. 1-112v) Epiphanius von Salamis, Panarion (Adversus Haereses), Kap. 65-80

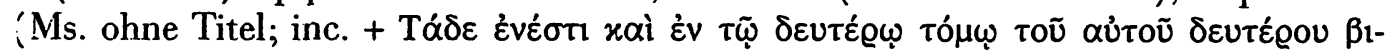

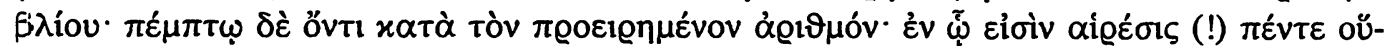
$\tau \omega \varsigma$.)

Epiphanius Ancoratus und Panarion. Dritter Band, ed. K. Holl/J. Dummer [GCSS 37] (Berlin -1985) 1-496 (= CPG 3745).

- Eine sechste (Ms. G.B. q: 5, s. XVII/XVIII) mit.den Fabeln Aesops (vgl. Corpus Fabularum Aesopicarum. Vol. I ed. August Hausrath. fasc. $1^{2}$ ed. Herbert Hunger [Leipzig ${ }^{+1970]}$ XVI Nr. 79 Jenensis Goethianus 5 saec. XVII-XVIII fab. 130 - Goeth. cont. Hsr., gehörig zur Rezension III $\beta \Lambda$ ) a urde zusammen mit dem größeren Teil der Bibliothek Wolfgang Maximilian von Goethes bei der Zerstörung des Hauptgebäuđess der Universitätsbibliothek durch einen Bombentreffer am 19.2. 1945 vernichtet.

${ }^{7}$ Diese Signatur erhielt die Handschrift allerdings erst 1968 (vgl. die handschriftliche Eintragung im maschinenschriftlichen Katalog "Griechische Handschriften der Universitätsbibliothek Jena- aus dem Jahr 1957): die falsche Zuordnung liegt eventuell darin begründet, daß bei der Zerstörung des Hauptgebäudes der Universitätsbibliothek in Jahr 1945 der Katalog der GoetheBibliothek verloren ging.

${ }^{8}$ Allgemeine Deutsche Biographie 10, 1879, 38-40; Günther, Lebensskizzen (wie Anm. 3), 130: Deutsche biographische Enzyklopädie 4 (1996) 225.

'Die Handschrift wird im Catalogus Bibliothecae Chr. Gottfr. Cruner, Pars I, Libri Medici, Jena 1818 auf S. 361 unten Nr. 5831 angefülırt: „Ein altes griechisches Msc: welches sich anfängt tov

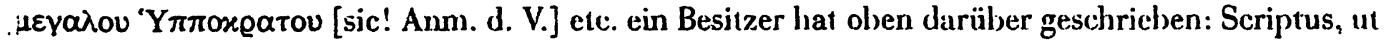
videtur, circiter 1530."

10 Alggekürzt zitierte Litcratur: Clavis Patrum Graecorum. Volumen Il Ab Athanasio ad Chrysostomurn. ed. M. Geerard. Turnhout 1974 (= CPC); Julien Leroy, Les types de reglure des manuscrits Grecs, Paris 1976 (= Leroy): C. M. Briquet, Les filigrancs. Dictionnaire histori(jue des marques du papier dès leur apparition vers 1282 jusqu'en 1600, Bd. 1-4, Leipzig ${ }^{2} 1923$ (= Bricjuet); E. Camillscheg/D. Harlfinger/H. Hunger, Repertorium der gricchischen Kopisten 800-1600. 1. Teil: Handschriften aus Bibliotheken Großbritanniens, Wien 1981 (= Repertorium I); E. Gamillscheg/D. Harlfinger/H. Hunger, Repertorium der griechischen Kopisten 800-1600. 2. Teil: i. landschrifien aus Bibliotheken Frankreichs und Nachı̈räge zu den Bibliothıken Croßbritunniens, Wien 1989 (= Reperworium II); E. Gamillscheg/D. Harlfinger/H. Hunger (u. Mitarb. v. P. Eleuteri), Repertorium der gritchischen Kopisten 800-1600. 3. Teil: Handschrifien aus Biblicotheken Roms mit dem Vatikan. Wien 1997 (= Repertorium III). 


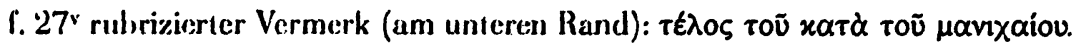

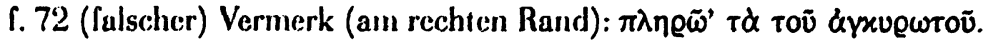

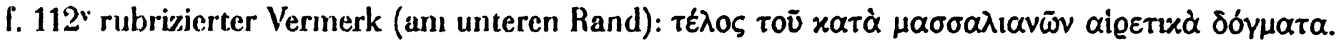

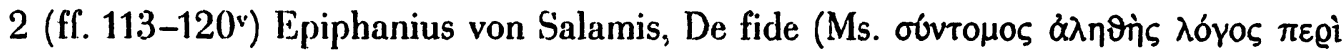

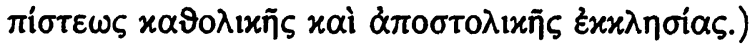

Epiphanius Ancoratus und Panarion. Dritter Band, ed. K. Holl/J. Dummer [GCS 37] (Berlin 21985$)$ 496-526.

f. 120" Der Text läuft in einem auf der Spitze stchenden Dreieck aus; darunter Subscriptio: $\tau \hat{\lambda} \lambda o \varsigma$

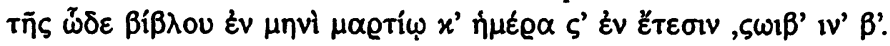

3 (f. 121) Kurzbiographie des Epiphanius von Salamis (Ms. ohne Titel, inc. ó จะĩos

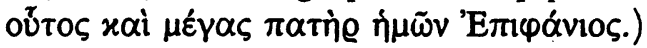

Epiphanius Ancoratus und Panarion. Erster Band, ed. K. Holl [GCS 25] (Leipzig 1915) 1.

4 (ff. 121-152v) Epiphanius von Salamis, Ancoratus (Ms. ohne Titel, inc. Ė

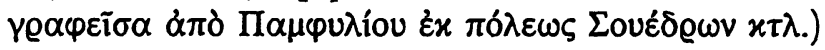

Epiphanius Ancoratus und Panarion. Erster Band, ed. K. Holl [GCS 25] (Leipzig 1915) 2-149 (= CPG 3744).

5 (ff. 152v-161) (Ps.-)Epiphanius von Salamis, Anacephalaeosis (Ms. ohne Titel, inc.

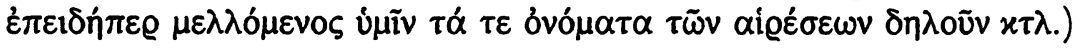

PG 42,833-885 (= CPG 3765).

6 (ff. 161-168) Epiphanius von Salamis, De mensuribus et ponderibus (Ms. olıne Ti-

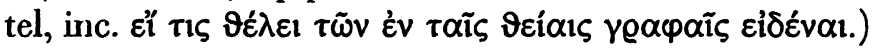

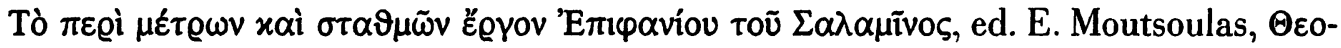

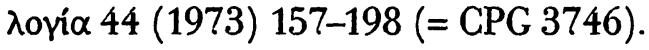

\section{7 (ff. 168-168v) Zitate aus (Ps.-)Johannes Chrysostomus}

(a) Johannes Chrysostomus, De incomprehensibilitate Dei 5 (Ms. Tit. тoũ ह̇v áyíos

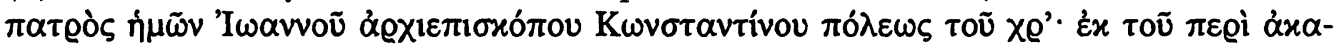

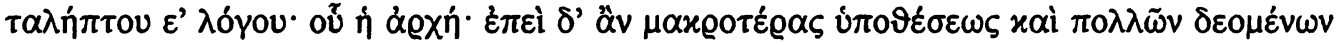

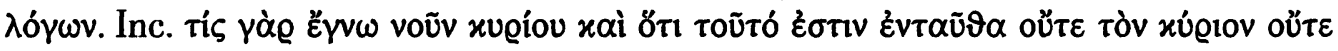

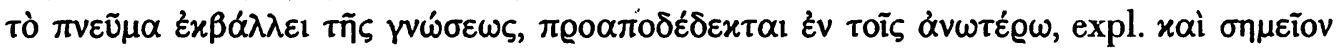

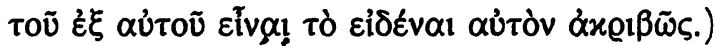

Jean Chrysostome. Sur l'incompréhensibilité de Dieu, ed. A. M. Malingrey [Sources Chrétiennes 28 ${ }^{\text {his }}$ ] (Paris 1970) 290,233-292,249 (= CPG 4318).

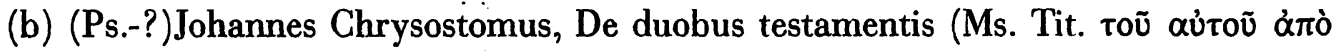

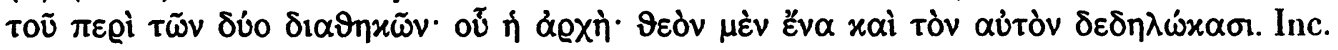

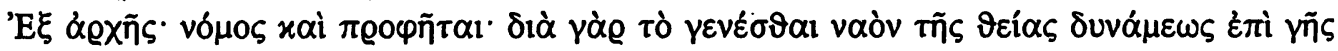

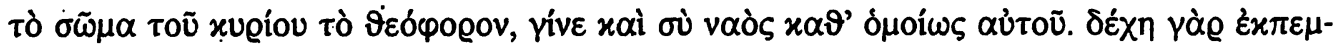

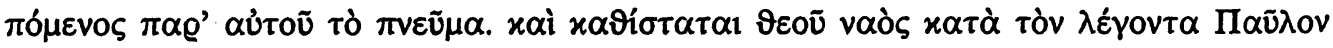

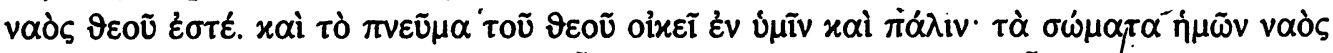

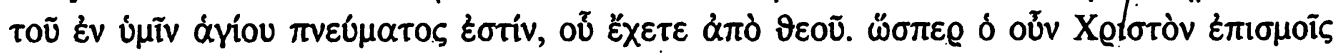

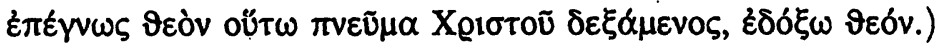

Ineditum

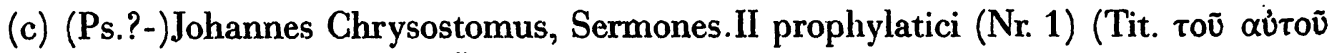

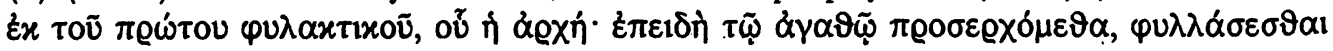




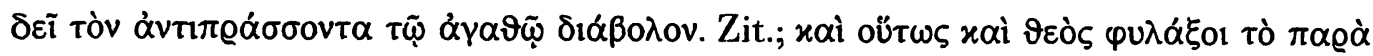

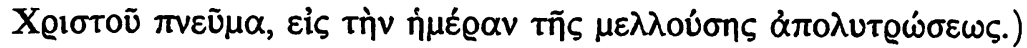

Ineditum (= CPG 4912).

(d) Ps.-Johannes Chrysostomus (= Severian von Gabala), In illud Abrahae

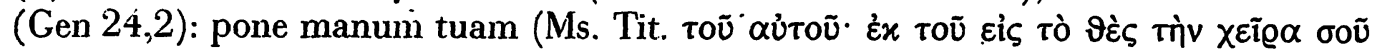

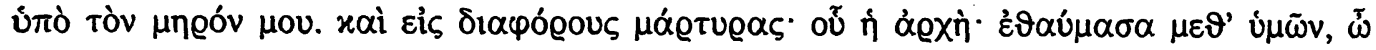

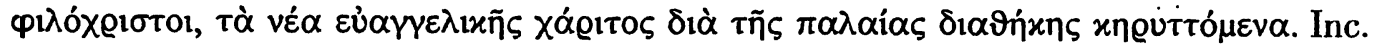

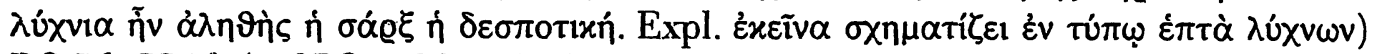
PG 56, 554f. (= CPG 4198; J. A. de Aldama, Repertorium Pseudo-Chrysostomicum, Paris 1965, Nr. 80).

(e) (Ps.-)Johannes Chrysostomus (= Severian von Gabala), In illud Abrahae

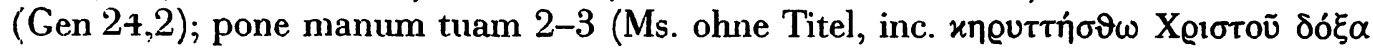

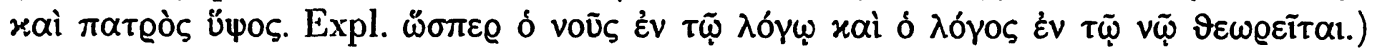
PG 56, 555f. (= CPG 4198; J. A. de Aldama, Repertorium Pseudo-Chrysostomicum, Paris 1965, Nr. 80).

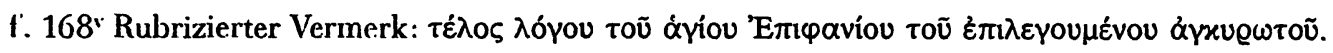

8 (ff. 169-174) Nicephorus von Konstantinopel, Chronographikon syntomon (Ms.

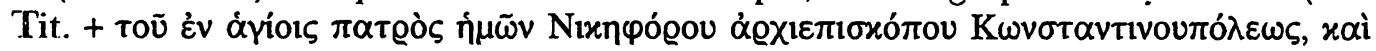

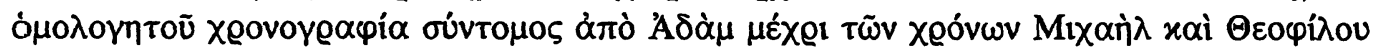
$+++)$

Nicephori archiepiscopi Constantinopol. opuscula historica, ed. C. de Boor (Leipzig 1880) 81-135.

Leer: f. 174́".

Textüberlieferung. Vgl. Holl (s. u. Bibl.), 76-87.

Foliierung. Durchgehende Foliierung am rechten oberen Rand; daneben ist gelegentlich noch eine ältere Foliierung zu erkennen, die jedoch größtenteils abgeschnitten ist.

Lagen. (ff. 1-120) $15 \times 8$, (ff. 121-168v) $6 \times 8$, (ff. 169-174v) $1 \times 6$.

Kustoden. Griechische Kustoden $\alpha^{\prime}-1 \varepsilon^{\prime}$ (ff. 1-120v) und $\alpha^{\prime}-\varsigma^{\prime}$ (f. 121-168v) auf der Recto-Seite des ersten Blattes der jeweiligen Lage rechts unten; Reklamanten auf dem jeweils letzten Blatt der Lage ebenfalls rechts unten.

Die ff. 169-174' sind deutlich nachträglich an den Buchblock angebunden (vgl. auch unten Erhaltungszustand) und tragen keine Kustoden.

Beschreibstoff. Bombyzin.

Schreiber. (ff. 1-120v) Hand A (vgl. Abbildung 1), datiert durch die Subscriptio auf 'f. 120' auf den 20. März 1304. Senkrechte bis leicht rechtsgeneigte Schrift mit Elementen des Fettaugenstils. Die ff. 1 u. $120^{v}$ sind besonders sorgfältig ausgearbeitet. An den Zeilenanfängen bisweilen vergrößerte Buchstaben; verhältnismäßig kürzungsreich. übèrgroße Abkürzung für - $\omega v$.

(ff. 121-168v) Hand B um 1300, höchstwahrscheinlich mit Hand $\dot{A}$ identisch (vgl. Abbildung 2).

Hand A und B: Überschriften in rubrizierter alcxandrinischer Auszeichnungsmạiuskel. Initialen und Versalien in rubrizierter epigraphischer Auszeichnungsmajuskel.

(ff. 169-174) Hand C (vgl. Abbildung 3), zu datieren in das zweite Viertel des 14. Jh.s. Senkrechte bis leicht rechts geneigte Schrift.

Lateinische und griechische Marginalien von Philipp Melanchthon (f: 2; 5; 11"; 16; 
$\left.18 ; 31^{v} ; 32 ; 54^{v} ; 55 ; 74 ; 108 ; 108^{v} ; 115^{v} ; 116 ; 118 ; 119 ; 120 ; 158^{v} ; 160 ; 164^{v}\right)$ : griechische und lateinische Marginalien (Titel der einzelnen Schriften) von einer Hand des 18.(?) Jh.s (f. 1; 51' ; 92v ; 113; 121; 152v; 161; 168v); griechisch-lateinische Marginalie von einer Hand des 16.(?) Jh.s (f. 58) (Edition dieser Marginalien bei Dummer/Markschies, s. u. Bibl.).; griechische Marginalie von Hand des 15.(?) Jh.s (f. 73 und 74"); griechische Marginalien von einer weiteren Hand (f. 152 und f. 166").

Am äußcren Rand finden sich außerdem häufig mit Silberstift geschriebene Zahlenangaben, die von der Herstellung der editio princeps der Werke des Epiphanius herrühren (s. u. Prov.).

Provenienz. Auf f. 1 befindet sich der Besitzvermerk „Ex bibliotheca Johannis Lang. Erphurdien.“. Demnach war die Handschrift einst im Besitz des Reformators von Erfurt, Johannes Lang (1487-1548) ${ }^{11}$. (Direkter?) Vorbesitzer war Johannes Trithemius, wie sich aus dessen Bücherverzeichnis erschließen läßt (vgl. Lehmann. s. u. $B i b l$.). Der Codex ist der zweite Band einer ehemals zweibändigen Handschrift. Diese diente über Vermittlung Philipp Melanchthons als Vorlage für die 1543 in Basel erschienene lateinische Übersetzung durch Janus Cornarius (vgl. Verzeichnis der im deutschen Sprachbereich erschienenen Drucke des 16. Jahrhunderts, hrsg. von der Bayerischen Staatsbibliothek in München in Verbindung mit der Herzog August Bibliothek in Wolfenbüttel, Bd. 6 (Stuttgart 1986) [= VD 16] E 1644) und für die 1544 gedruckte griechische Editio princeps durch Johannes Oporinus (vgl. VD 16, E 1643). Von dieser Benutzung als Druckvorlage zeugen an mehreren Stellen in der Handschrift Fingerabdrücke mit Druckerschwärze und Zahlenangaben im Text. Der erste Band der Handschrift ist (wohl zwischen 1612 und 1676) verlorengegangen. 1676 gelangte der heute noch vorhandene Band aus dem Nachlaß von Johann Andreas Bose in die Universitätsbibliothek. Dieser hatte ihn oder die noch vollständige Handschrift seinerseits wohl von Kaspar von Barth ( +1658 in Leipzig) erworben, in dessen Besitz die beiden Epiphanius-Bände seit dem Jahr 1612 waren (vgl. dazu Holl, 4, s. u. Bibl.).

Lin. 46 (ff. 1-168v), 44 (ff. 169-174v). Schriftspiegel $242 \times 156 \mathrm{~mm}$. Tinte: (ff. 1-168v) dunkelbraun, Rubriken rot; (ff. 169-174v) helles Braun, Rubriken rot.

Illustrationen. Federproben: f. 31, 85, 91 (Kreis/Zirkelzeichnung); f. 85" (Kreis). f. 1 rubrizierte, intermittierende Ranke mit Halbpalmetten auf dunklem Grund in Rahmen mit Knospen, Eckausläufern und Palmette mittig auf dem oberen Rand. f. 1; $28 ; 29^{v} ; 51^{v} ; 69 ; 25 ; 110 ; 121$ rubrizierte Leiste mit drei Spangen, die in Dreiblätter ausläuft. f. $29^{v} ; 63$ in Dreiblätter auslaufende, durch Text unterbrochene rubrizierte Leiste. f. 4; 32v ; 57; 59vi $152^{v}$; 168 in Kleeblätter auslaufende rubrizierte Fadenranke mit alternierenden linearen Elementen. f. 56 rubriziertes, in Dreiblatt auslaufendes streifenförmiges Flechtornament mit Rahmen; dasselbe, aber ohne Rahmen f. 73;

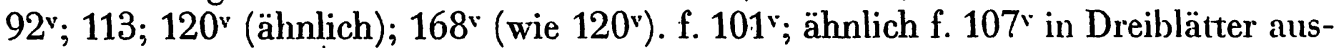
laufende, unterbrochene rubrizierte Leiste. $f .115 ; 115^{\mathrm{v}}$ in roter Tinte ausgeführte Unterstreichungen im Text.

Einband. Holzband in Leder gebunden mit spätgotischer Streicheisenverzierung und Blindstempelung. Der Band wurde bei der Restaurierung des Codex im Oktober 1974

11 Vgl. Allgemeine Deutsche Biographie 17 (1883) 635-637; R. Stupperich, Art. Lang(e), Johannes, Neue Deutsche Biographie 13 (1982) $540 \mathrm{f}$; Deutsche biographische Enzyklopädie 6 (1997) 226. 
unter Verwendung des alten Materials neu gebunden. Am Vorderschnitt sind zwei, jedoch nicht mehr lesbare Buchstaben zu erkennen.

Erhaltungszustand. Bei einer Restaurierung im Oktober 1974 wurden vorhandene Schäden (Feuchtigkeitseinwirkungen, Papierfaserzerfall und Tumorbildung) weitgehend beseitigt.

(ff. 169-17+ $4^{v}$ ) Wurmfraß.

\section{Bibliographie.}

Nicephori archiepiscopi Constantinopolitani opuscula historica, ed. C. de Boor (Leipzig 1880) XLNf.

K. Holl, Die handschriftliche Überlieferung des Epiphanius [TU 36/2] (Leipzig 1910) 1-5.75-80.

P. Lehmann, Merkwürdigkeiten des Abtes Johannes Trithemius, Sitzungsberichte der Bayerischen Akademie der Wissenschaften, phil.-hist. Kl., Jg. 1961, Heft 2 (München 1961) $10 \mathrm{f} .30 \mathrm{f}$.

J. Dummer/Ch. Markschies, Philipp Melanchthons Randbemerkungen zu Epiphanius im Jenensis Bos. f. 1 (zum Druck vorbereitet).

\section{Bos. q. 7}

Sophokles mit Scholien/„Versspielereien“

Letztes Viertel 15. Jh. Papier I, 184, II' $225 \times 160 \mathrm{~mm}$

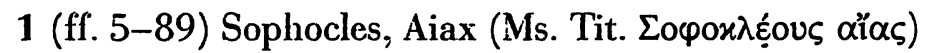

Sophoclis Fabulae, ed. H. Lloyd-Jones/N. G. Wilson (Oxford 1990)-3-57.

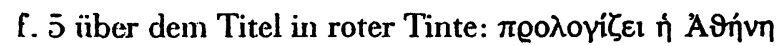

f. $89^{\mathrm{r}}$ leer

\section{vorausgehend}

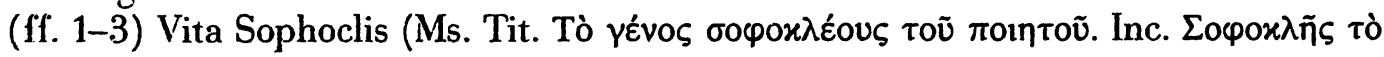

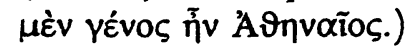

Sophoclis Electra. Editio tertia curata ab Adolpho Michaelis, ed. O. Jahn (Bonn 1882) 1-15.

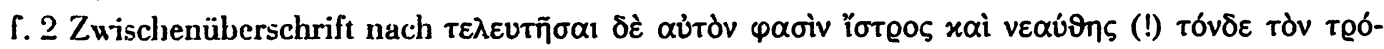

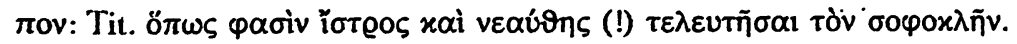

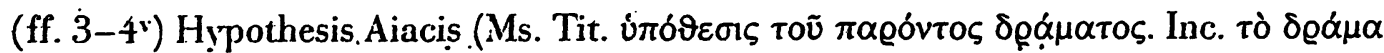

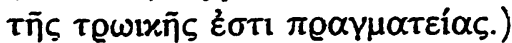

'Sophocles. The Plays and Fragments. Part VII. The Aiax, ed. R. C. Jebb (Cambridge $1907=$. ID Amsterdam 1967) 3-6.

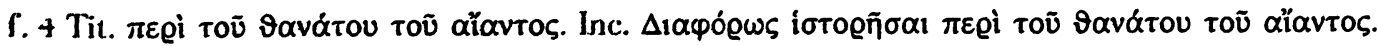

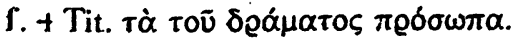

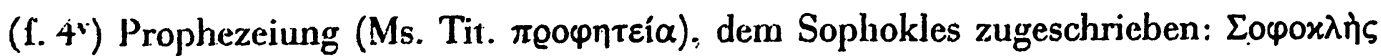

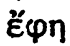

Thersophorum Craecorum Fragmenta, iterum recensuit H. Erbse (Stuttgart/Leipzig $1995=) 113$.

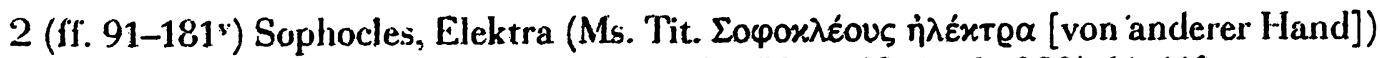
Sophoclis Fabulac, ed. H. Lloyd-Jones/N. G. Wilson (Oxford 1990) 61-118. 


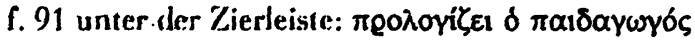

vorausgehend

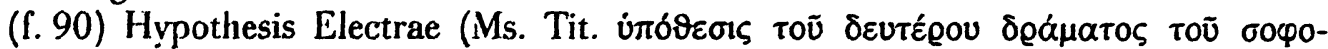

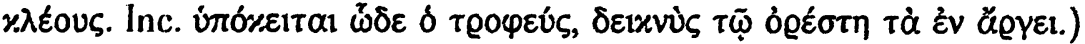

Sophocles. The Plays and Fragments. Part VI. The Electra, ed. R. C. Jebb (Cambridge 1924) $3 \mathrm{f}$.

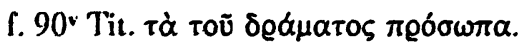

(f. 5-89; 91-181 ${ }^{\mathrm{v}}$ ) Interlinearglossen von der Hand des Schreibers

( . 5-89: 91-181") in marg. von derselben Hand Scholien (ed. L. Purgold, Observationes criticae [s. u. Bibl.], 61-116. 147-203)

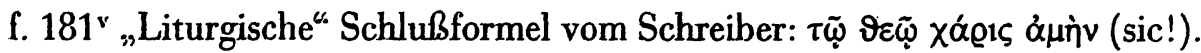

3 (f. 182-182v) Von anderer, zeitgleicher Hand verschiedene „Versspielereien*

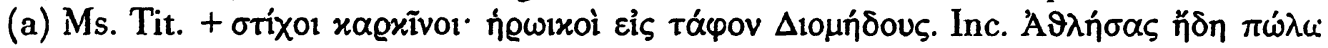

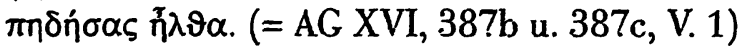

Anthologie grecque. Deuxième Partie. Anthologie de Planude, Tome XIII, ed. R. Aubreton/F. Buffière, (Paris 1980) 227.

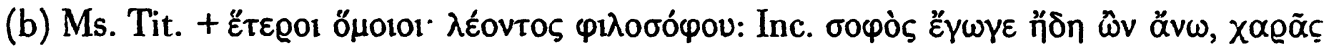
$\tau \tilde{\omega} \nu \not ̈ \nu \omega, \gamma \varepsilon \lambda \tilde{\omega} \tau \grave{\alpha}$ xó่ $\tau \omega$.

Excerpta varia Graecorum Sophistarum, ed. L. Allatius (Rom 1641) 398 (= PG 107. $665 \mathrm{f}$.); jedoch fehlen die dort abgedruckten letzten vier Verse)

(c) 3 Verse

1. Vers mit allen acht Wortarten

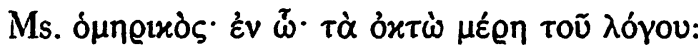

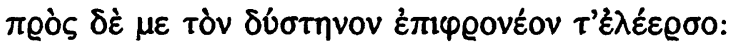

2. Vers mit allen Buchstaben, Matthaios Chrysokephalos zugeschrieben

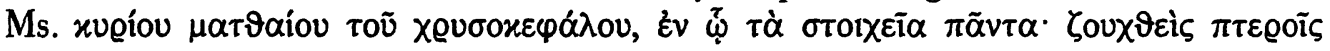

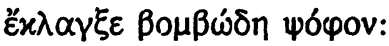

Dieser Vers ist, anonym, auch im Cod. Par. gr. 3058 überliefert.

3. Anonymer Vers mit allen Buchstaben (in Cod. Par. gr. 3058 dem Johannes Tzetzes zugeschrieben)

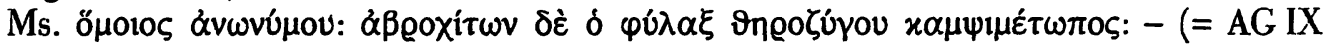
538).

Anthologie grecque. Première Partie. Anthologie Palatine. Tome VIII (Livre IX, Épigr. 359-827), ed. P. Waltz/G. Soury (Paris 1974), 80.

Leer: f. $89^{v}$.

Textüberlieferung. Vgl. Turyn; Petrocione (s. u. Bibl.).

Foliierung. 1-91, 91b (mit Bleistift noch 92! 93!), 92-183.

Lagen. $23 \times 8$. Kustoden. Die Kustoden der ersten beiden Lagen fehlen; danach Kustoden von $\gamma^{\prime}-x \gamma^{\prime}$
am rechten unteren Blattrand.

Beschreibstoff. Papier.

Wasserzeichen. (ff. 1-16) Waage ähnlich Briquet 2597 (Venedig 1490) u. Briquet 2598 (Venedig 1499, Gegenzeichen aber ohnc ; $b^{\text {"s) }}$; (ff. 17-24) Hut (kein Vergleichsstück); (ff. 25-32) Turm (kein Vergleichsstück); (ff. 41-48) Hut; (ff. 50/55) Hut; 
(ff. 51/54) Waage; (ff. 57-72) Waage; (ff. 73-183) Armbrust ähnlich Piccard IX/2, 2229 (Casola Valsenio 1491).

Schreiber: (f. 1-181") 〈Johannes〉 (Harlfinger, auf Grund des Duktus [vgl. Abbildung 4]), vgl. Repertorium I, Nr. 203; II, Nr. 279; III, Nr. 336; von ihm stammt auch Codex Parisinus gr. 2598 (datiert 11. 7. 1467), dessen Sophokles-Text ebenfalls der „Jena Recension" (Turyn) angehört. Illustrationen und Interlinearglossen sind in hellroter Tinte ausgeführt; der Text in schwarzbrauner Tinte.

(f. 182-182 ) von anderer, vermutlich zeitgleicher rechts geneigter Hand.

Provenienz. Die Handschrift stammt aus der Bibliothek Boses, wie der Besitzvermerk "Jo. Andrea Bosii" auf dem Vorsatzblatt zeigt. Bose hatte die Handschrift wohl in Leipzig erworben. Auf f. 1 findet sich der Besitzvermerk „Ex bibliotheca Fratris M. Jacobj. M. Guil. Schmugck.". Jakob Schmuck"12 aus Sull war nach dem Studium in Leipzig als Rektor des Gymnasiums in Schleusingen tätig; er verstarb aber bereits $1599 \mathrm{im}$ Alter von nur 28 Jahren; sein um vier Jahre jüngerer Bruder Wilhelm ${ }^{13}$, der die Handschrift erbte, lehrte bis zu seinem Tod 1634 als Professor beider Rechte an der Universität Leipzig. Vgl. Handschriften und alte Drucke, 6 f. (s. u. Bibl.).

Lin. 24 (ff. 1-4v); 8 (+ Interlineargl.; Randscholien max. 35) (ff. 5-181). Schriftspiegel: $134 \times 80 \mathrm{~mm}$ (Haupttext).

Illustrationen. f. 1 Band in Dreiblatt mit Knospen auslaufend; f. 5 intermittierende Ranke mit Dreiblättern auf rotem Grund; f. 90 intermittierende Ranke mit Halbpalmetten auf rotem Grund; f. 91 spiegelsymmetrische Palmettenranke auf rotem Grund in Rahmen mit Eckausläufern. f. 1 Rankeninitialie; f. 5 Initiale $A$ in epigraphischer Auszeichnungsmajuskel mit Ranken; f. 90 Initiale $Y$ in epigraphischer Auszeichnungsmajuskel mit Ranke; f. 91 Initiale $\Omega$ als gekrönter Doppeladler. Versalien in epigraphischer Auszeichnungsmajuskel.

Einband. Pergamentüberzogener Pappband, durch Streicheisenlinien gegliedert und mit einigen floralen Elementen verziert; auf dem Vorderdeckel eingeprägte Buchstaben „P S S“ und die Jahreszahl „1598“, auf dem Rückdeckel „M I S“ (d. i. Magister Jakob Schmuck); Goldschnitt.

Erhaltungszustand. Geringe Wasserflecken an den Blatträndern.

Bibliographie.

L. Purgold, Observationes criticae in Sophoclem, Euripidem, Anthologiam Graecam et Ciceronem; adiuncta est e Sophoclis codice Ienensi varietas lectionum et scholia maximam partem inedita '(Jena/Leipzig 1802) 3-9 (allg.; Sophokles). 277-279 (Versspielereien).

A. Turyn. The Manuscripts of Sophocles, Traditio 2 (1944) 19-20. ders., Studies in the Manuscript Tradition of the Tragedies of Sophocles [lllinois Studies in Language and Literature, 26] (Urbana 1952) 87-95.

R. D. Dawe, Studies on the Text of Sophocles I (Leiden 1973) 138.196.

J. Petrucione, The Text of Sophocles' Ajax in the Codex Ienensis (Bos. q. 7), Zeitschrift für Papyrologie und Epigraphik 53 (1983) 37-52.

Sophoclis Fabulae, ed. H. Lloyd-Jones/N. G. Wilson (Oxford 1990) XII.

12 Vgl. Jöcher, Allgeıneines Gelehrten-Lexicon (wie Arm. 3) IV (Leipzig 1751) 308.

13 Vgl. Jöcher, Allgemeines Celehrten-Lexicon (wie Anm. 3) IV $308 \mathrm{f}$. 
Handschriften und alte Drucke. Texte antiker Schulautoren, ausgewählt und erläuterı von G. Huber-Rebenich/I. Kratzsch (Jena 1996) $6 \mathrm{f}$.

\author{
G.B. q. $6^{\mathrm{a}}$
}

Typikon des Klosters Nea Odigitria von Rossano (gen. Patir)

Zw. 8. 7. 1152 u. 19.7. $1182 \quad$ Pergament ff. I, 189, I' $243 \times 170 \mathrm{~mm}$

1 (ff. 1-8) Kephalaia

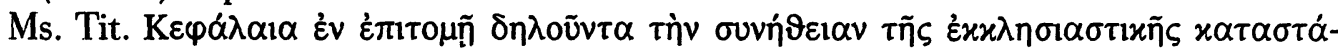

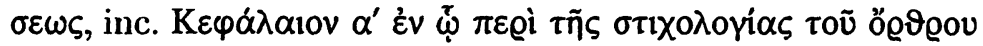

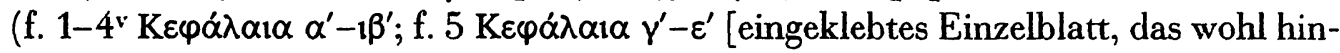

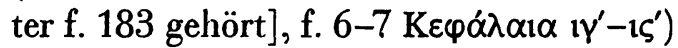

f. 2 Notiz an unteren Rand von einer anderen Hand (ed. S. G. Mercati, Sulle Reliquie [s. u. Bibl.], 407.

2 (ff. $8^{\mathrm{r}}-104^{\mathrm{v}}$ ) Synaxarion

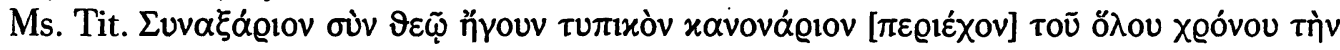

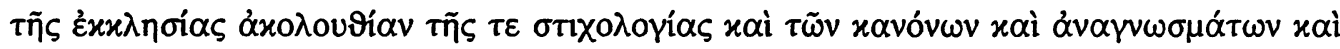

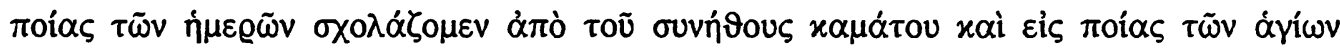

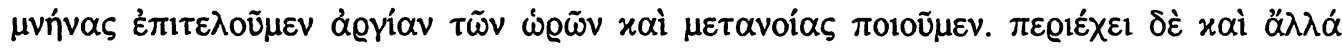

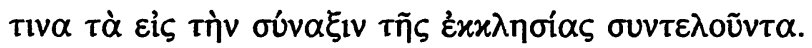

[f. $8^{\mathrm{r}}$ September; f. 21 Oktober; f. 29 November; f. 42 Dezember; f. 57" Januar (von späterer Hand nachgetragen oder nachgezogen); f. 73" Februar; f. 77 März; f. 79v April; f. 81 Mai; f. 83v Juni; f. 88 Juli; f. 95 August]

f. $39^{*}$ Marginale Notiz von einer anderen Hand über den Tod des Hegumenos Niphon im Jahr 1252 (vgl. S. G. Mercati, Sulle reliquie, 407)

f. 100-101 Notiz über den Tod des Klostergründers Bartholomäus am 19. August 1130: $\mu \eta v i ̀ ~ \tau \tilde{\varphi}$

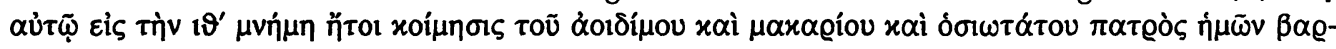

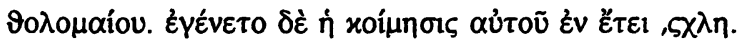

3 (ff. 105-160v) Triodion - Pentekostarion

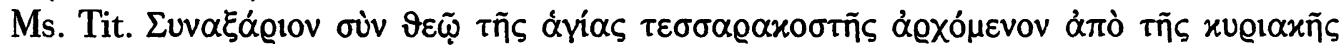

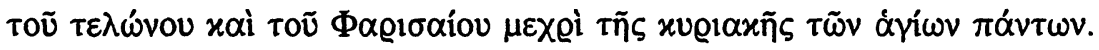

f. $118^{v}$ Tit. $\gamma v \tilde{\omega} \sigma i \varsigma \tau \tilde{\omega} v 1 \delta^{\prime} \delta \varepsilon \sigma \pi \circ \tau x \tilde{\omega} v \dot{\varepsilon}$

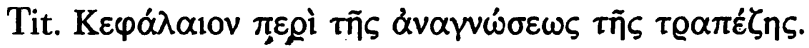

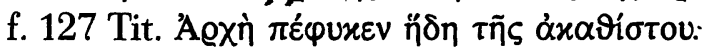

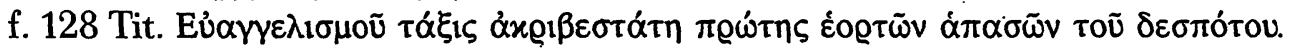

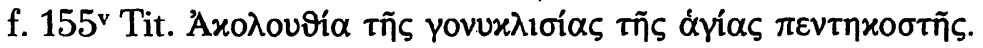

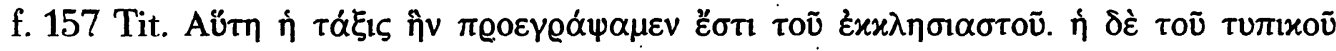

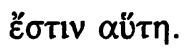

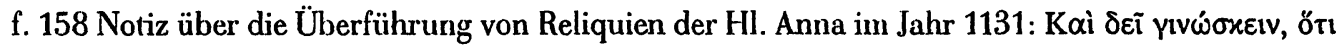

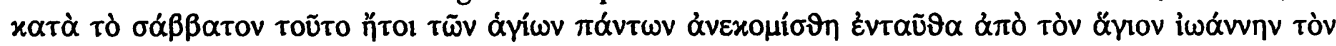

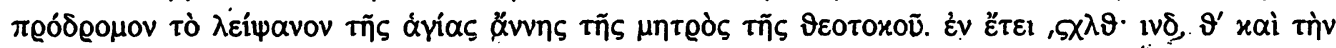

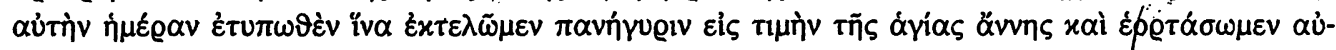

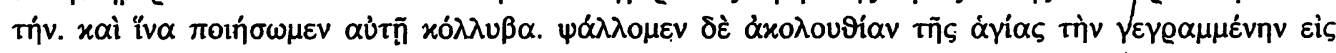

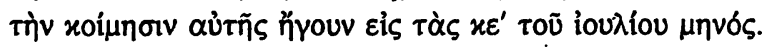

5 (ff. 161-170) Ktetor-Typikon des Bartholomäus

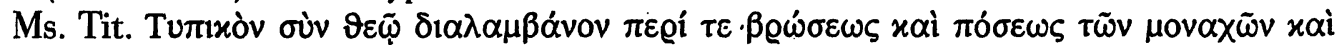

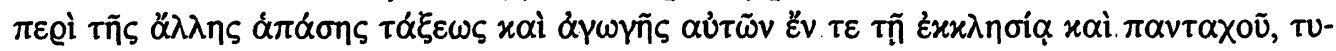




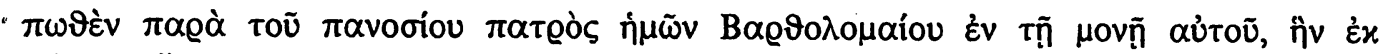

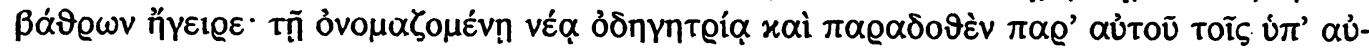

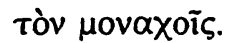

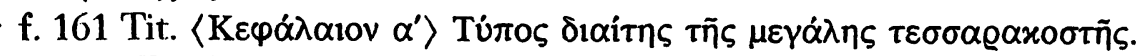

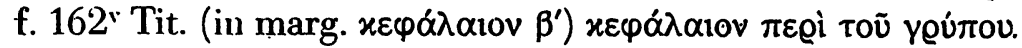

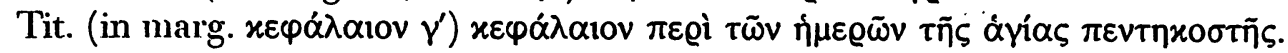

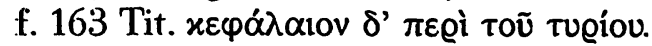

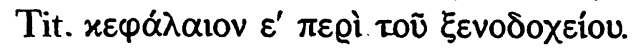

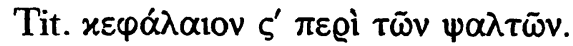

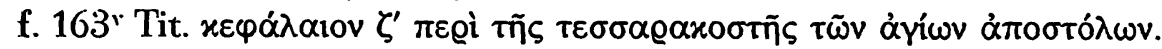

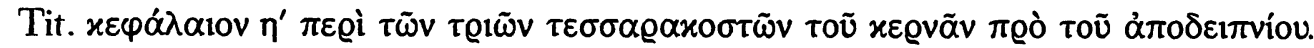

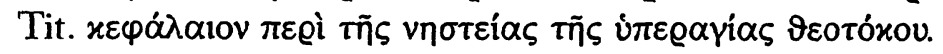

f. 164 Tit. (in marg. $x \varepsilon \varphi \alpha ́ \lambda \alpha 10 v \imath^{\prime}$ ) $\pi \varepsilon \varrho i ̀ ~ x o \lambda \lambda u ́ \beta \omega v$.

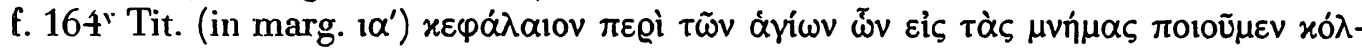
$\lambda v \beta \alpha$.

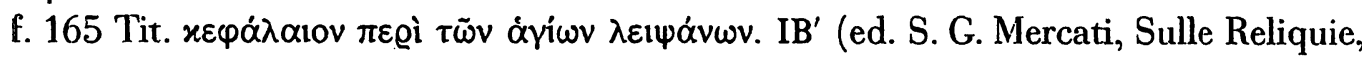
396-401)

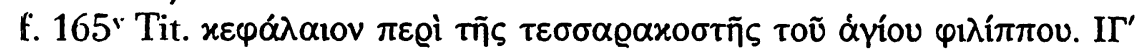

f. 166 Tit. $x \varepsilon \varphi \alpha ́ \lambda \alpha 10 v \pi \varepsilon \varrho i ̀ ~ \tau \tilde{\omega} v \pi \varepsilon \lambda \varepsilon x \alpha v \tilde{\omega} v . \mathrm{I} \Delta^{\prime}$

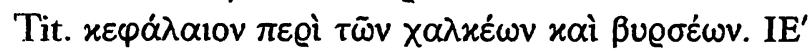

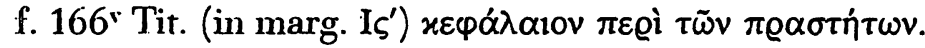

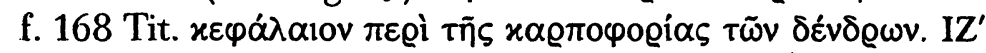

Tit. (in marg.) $\pi \varepsilon \varrho i ̀ ~ \tau \tilde{\omega} v$ ỏ

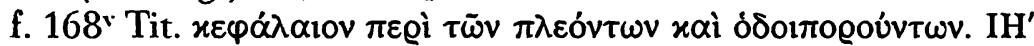

f. 169 Tit. $x \varepsilon \varphi \alpha \dot{\lambda \alpha 10 v} 1 \vartheta^{\prime} \pi \varepsilon \varrho i ̀ ~ \pi \varepsilon x o u \lambda i ́ \omega v$.

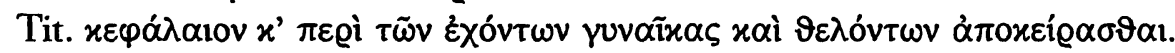

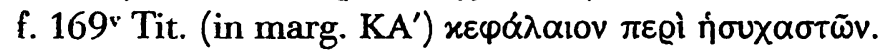

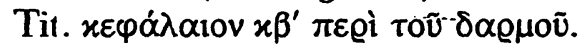

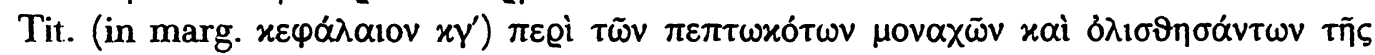

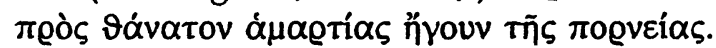

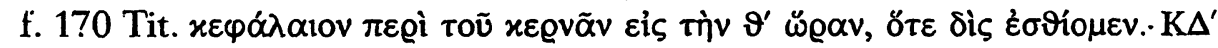

6 (ff. 170-189) Ergänzungen zum Ktetor-Typikon

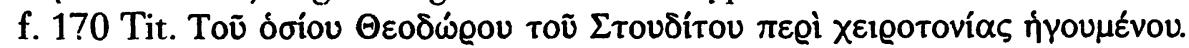

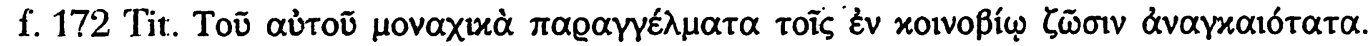

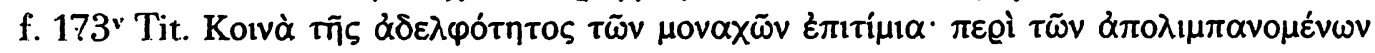

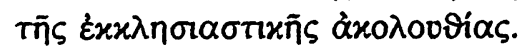

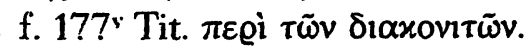

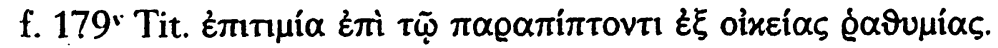

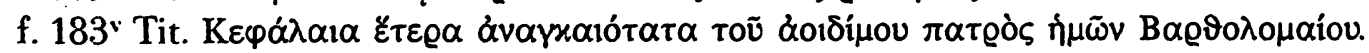
(hierher f. 5)

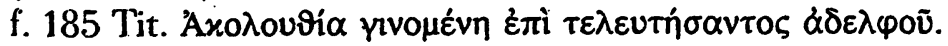

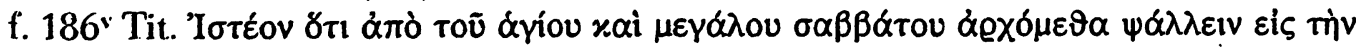

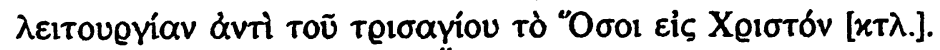

f. $187^{r-v}$ Notizen über die Überführung von Reliquien der 40 Märtyrer (von Sebaste) und der 63 Megalomärtyrer am 31. 1.1109, des Apolinaris am 16. 11. 1112 und des Prokopios, Aberkios, Merkurios, Fortunatus und Bartholomäus am 8. 7. 1152 von der Haupthand (ed. S. C. Mercati, Sulle Reliquie, 401 f. 404).

f. 187' Notiz über die Überführung von Reliquien des Johanncs Elemosynarius, des 
Blasius und des Menas am 19.7. 1182 von einer weiteren Hand (ed. S. G. Mercati, Sulle Reliquie, 404).

Notiz über die Überführung von Reliquien des Kaisarios, Julianus und der Eugenia „im zweiten Jahr nach dem Tod Wilhelms II. von Sizilien" (†16.11. 1189) von einer weiteren Hand (ed. S. G. Mercati, Sulle Reliquie, 405).

Notiz über den Tod des Abtes Chariton am 7.6.1269, von dem das Kloster eine Haarreliquie der Muttergottes erhalten hatte, von einer weiteren Hand (ed. S. G. Mercati, Sulle Reliquie, 405).

Notiz über die Überführung von Reliquien des Silvester von Rom, des Therapon, des Philaretos, des Hilarius, des Konon, des Alphios und Philadelphos und der Uliva am 2. 9. 1303 von einer weiteren Hand (ed. S. G. Mercati, Sulle Reliquie, 406).

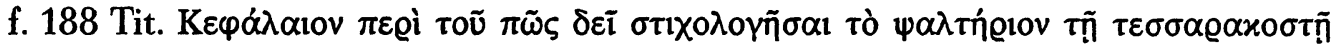

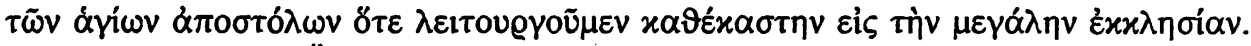

f. 188" Notiz über Überführung von Reliquien des Johannes Chrysostomos im Jahr 1293 von einer weiteren Hand (ed. S. G. Mercati, Sulle Reliquie, 401).

f. 189 Notizen verschiedener Hände:

I. Notiz über den Tod des Mönches Jakobos im Jahr 1548.

II. (Lateinische) Notiz über den Tod des Priors Martinus am 27. August 1523.

III. Nicht mehr lesbar.

IV. Notiz über Überführung von Reliquien der Hl. Kallinike im Jahr 1207.

\section{7 (f. 189v) Tafel zur Osterfestberechnung}

Ursprünglich der Pergamenthandschrift beigebunden, nach der modernen Restaurierung in Schuber als Einzellagen beigegeben:

1 (ff. 190-219) Lateinische Übersetzung der ff. 161-183 der Pergament-Handschrift

Ms. Tit. Typicum Dei gratia complectens ea, quae spectant ad cibum, et potum Monachorum ... praescriptum a SS. P. N. Bartholomeo in suo Monasterio ... appellaturque Nova Odighitria ... Ex Graeco in Latinum reddebatur. A. P. Magistro Abbate D. Theophilo ab Alexandro Procuratore Generali ordinis S. Basilii Ronae Anno 1712.

f. 203 Tit. Ex Divo Theodoro Studita Caput de electione et creatione Abbatis.

2 (ff. 220-222) Chronologische Zusammenstellung der verschiedenen Notizen über Reliquienüberführungen in lateinischer Übersetzung

Ms. Tit. Nonnullae adnotationes de reliquiis sacris sanctorum, quae translatae fuerunt ad praefatum monasterium SSae. Dei Genitricis Novae Odighitriae, et sparsim reperiuntur in suprad. Typico, hic vero descriptae juxta ordinem, et Seriem Annorum, videlicet.

3 (f. $222^{v}$ ) Lateiniśche Übersetzung von f. $8^{v}$ der Pergament-Handschrift

Ms. Tit. Synaxarium, seu Typicon regulare complectens Ecclesiae officia, stychologiam, canones et lectiones, quibusve diebus cessamus a consueto labore, quibusque ss. memoriis festum celebramus, et abstinemus horis, et metanias facimus. Complectitur quidem alia quae in Ecclesia peraguntur. Transcriptum saeculo ab Incarnatione Domini nostri Jesu Christi XII.

4 (ff. 223-224) Lateinische Übersetzung der Kephalaia von f. 1-8 sowie Inhaltsverzeichnis der Pergament-Handschrift

Tit. Index eorum quae in hoc Typico continentur.

Textüberlieferung. Auf f. 1 ist'am oberen Blattrand auf dem Kopf stehend' in Majuskel zu lesen:PSTH AГIH; sonst sind keine Buchstabenreste zu erkennen, die auf ein Palimpsest schließen ließen.

Eine Abschrift der Handschrift durch P. Sofronio Gassisi $(† 1923)$ und eine weitere Hand liegt in Codex Cryptensis 211 (olim Г..$\alpha$ XXIX) vor.

Foliierung. Alte Foliierung auf Recto des Blattes in der rechten oberen Ecke, mit Lük- 
ken noch sichtbar von 6-102. Neuere Foliierung von zwei Händen jeweils auf Recto des Blattes links unten und auf Verso links oben (teilw. doppelt) von 1-189. Von f. 161 bis f. 189 zusätzliche Foliierung von 1-28 auf dem Recto oben rechts vom Schreiber der Papierhandschrift; von f. 161-183 zusätzlich Foliierung in griechischen Buchstaben von $\alpha-x \gamma$ (mit Lücken) von der Haupthand; daneben von f. 164-185 zusätzliche Foliierung rechts oben von 172-192 (mit Lücken und Unregelmäßigkeiten: 172-174, [Lücke von einem Blatt], 176, 181-183, 186, [Lücke von zwei Blättern], 173, 187, [Lücke von zwvei Blättern], 190, 191, 185, 192.

Lagen. FHHF; $1 \times 2 ; 1 \times 4(+1 ;=$ f. 5$) ; 9 \times 8 ; 1 \times 10$ (f. 80 u. 88 Einzelblätter); $1 \times 6 ; 1 \times 10(-1) ; 1 \times 10(-1$; Blatt 1 fehlt $) ; 2 \times 8 ; 1 \times 6(-1$; Blatt 1 fehlt $)$; $1 \times 10 ; 1 \times 6 ; 1 \times 10 ; 2 \times 8 ; 1 \times 6 ; 1 \times 8(-1 ;$ nach f. 184). Keine Kustoden (mehr) vorhanden. Durch verschiedenfaches Binden ist die ursprüngliche Lagenzählung nicht mehr zu ermitteln. f. 88 ist (bei der Restaurierung) auf dem Kopf stehend eingebunden.

Beschreibstoff. Pergament unterschiedlicher Dicke und unregelmäßigen Zuschnittes.

Schreiber. Haupthand minuscola salentina (Lucà); alexandrinische Auszeichnungsınajuskel für Überschriften, epigraphische Auszeichnungsmajuskel für Initialen und Versalien.

Die Datierung der Haupthand ergibt sich aus dem auf $f .187^{\circ}$ feststellbaren Befund (vgl. Abbildung 5): Die zeitlich letzte Notiz der Haupthand über die Überführung von Reliquien bezieht sich auf eine Reliquienüberführung am 8. Juli 1152. Der nächste Eintrag über eine Reliquienüberführung am 19. Juli 1182 stammt bereits von einem von der Haupthand deutlich unterschiedenen Schreiber. Diesem Eintrag folgen von drei weiteren Händen in zeitlicher Abfolge Einträge über Reliquienüberführungen. Die Haupthand ist daher in den Zeitraum zwischen dem 8. Juli 1152 und dem 19. Juli 1182 zu datieren.-Die von Lucà, I Normanni (s. u. Bibl.), 11 Anm. 42 unter Verweis auf die (jedoch nicht von der Haupthand stammende) Notiz auf $f .2^{v}$ vorgenommene Datierung um die Mitte des 13. Jahrhunderts ist ebenso zurückzuweisen wie die auf derselben Notiz beruhende Datierung in den Zeitraum zwischen 1254 und $127 \&$ bei Jordanides, Typiko (s. u. Bibl.), 110.

Ergänzungen des Textes von verschiedenen Händen auf f. 5, 10, 12", 16", 21, 23, 24, $59,81,84^{v}, 101,104^{v} ; 106,116,117.165$.

Marginale Verweise auf andere Stellen im Typikon von drei weiteren Händen einge-

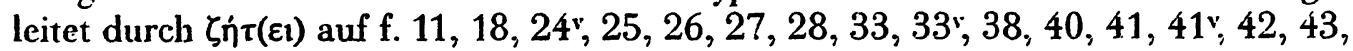
44. 58v $70.71,72,73^{v}, 77,78,79,80,80^{v}, 81,83,84,86,90,90 v, 91,91 v, 92,94$, $95,95 \% 97,100^{\mathrm{v}}, 102,103,150^{\mathrm{v}}, 153^{\mathrm{v}}, 158^{\mathrm{v}}$.

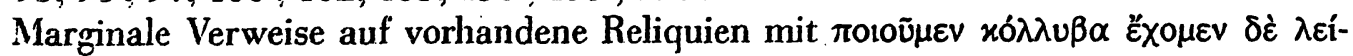

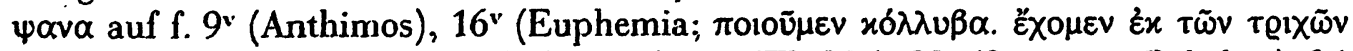
Tĩs áyías), 17 (Eustathios mit Gefährten), 18v (Thekla), 22v (Sergios u. Bakchos), 24 (Apostel u. Evangelist Lukas), 24v (Hilarion), 30 (Paulos Homologetes), 32 (Theodoros Studites), 32v (Johannes Elemosynarius), $40^{v}$ (Clemens von Rom), $42^{v}$ (Barbara), 46 (Eustratios mit Gefährten), 46* (Drei Jünglinge im Feuerofen und Daniel), 47* (Bonifatius), 48* (Ignatios Theophoros), 66 (Polyeuktos), 66" (Theodosios Koinobiarches), 72, 75 (Symeon Theodochos), 75" (Kallinke; Theodoros Stratelates), 76" (Blasios). 77 (Theodoros Tyron), 89v (Kyriake).

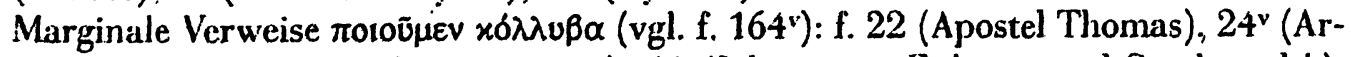
remios), 33 (Johannes Chrysostomos), 41 (Johannes v. Ephesos und Stephan d.J.), 
41. (Apostel Andreas), 43 (Sabas), 43v (Nikolaos), 45v (Menas, Hermogenes, Eugraphos; Spyridon Thaumaturgos), 46" (Eleutherios), 66 (Gregor von Nyssa). 69" (Euthymios d. Gr.), 71 (Gregor von Nazianz), 73 (Kyros und Johannes), 84 (Onuphrios), $84^{v}$ (Judas der Herrenbruder), 86v (Petros und Paulos), 93 (Maria Magdalena).

Weitere Marginalien auf f. 17v, 29, 32, 56, 59"; 89v, 102v, 115v, 117v, $158^{v}$.

Tinte teils pechschwarz, teils hellbraun. Überschriften in roter Tinte.

Provenienz. Es handelt sich um das Typikon des Klosters Nea Odigitria von Rossano (gen. Patir). Die Handschrift befand sich dort bis in die Mitte des 16. Jahrhunderts (vgl. die Notizen auf f. 189). Zu Beginn des 18. Jahrhunderts ist die Handschrift dann im Collegium S. Basilii in Rom nachweisbar (vgl. Lucà, I Normanni [s. u. Bibl.], 22 Anm. 89). Am 1. März 1846 wurde sie in Rom von Wolfgang Maximilian von Goethe aufgekauft, aus dessen Nachlaß sie in den Besitz der Universitätsbibliothek Jena kam. (Auf der Innenseite des Vorderdeckels war vor der Neubindung ein Zettel aufgeklebt mit folgendem Besitzvermerk: W. M. v. Goethe. | II.J.D.-| Rom. März. 1846. Dieser ist heute nicht mehr vorhanden; wahrscheinlich ging er bei der Restaurierung des Einbandes verloren. Allerdings befindet sich im Nachlaß von W. M. v. Goethe [Thüringer Universitäts- und Landesbibliothek, Nachlaß W. M. v. G., Mappe f. 40] ein Blatt mit Notizen von der Hand Goethes: „Manuscripte. In Rom kaufte ich am 1st. März 1846 Wolfgang Maximilian von Goethe II. I. d. ... Handschriften für 32 Scudi 18 Baj. [...] IV. Pergamenthandschrift aus dem 12.t. J. od. 13.t. Jahrhundert...".)

Linierung. (ff. 1-29) 20D1 Leroy, danach 00D1 Leroy. Lin. 22. Schriftspiegel: $173 \times 117 \mathrm{~mm}$.

Illustrationen. f. 1; 104 $4^{\mathrm{v}} ; 113 ; 118^{\mathrm{v}} ; 127 ; 135 ; 155^{\mathrm{v}} ; 157 ; 168 ; 183 ; 188$ in Kleeblatt auslaufende, durch senkrechte Striche in Dreigruppen unterbrochene Fadenranke (teils zum Auffüllen von Zeilen). f. $8^{v}$ Zierleiste mit Viererschlingen, Schrägkreuzmuster und Kreuzmuster in schwarzer und roter Tinte, mit Eckausläufern. f. 105; 135; $186^{\circ}$ Zierleiste in Dreiblatt auslaufend mit Knoten in schwarzer und roter Tinte. f. 161 rubrizierte, intermittierende Ranke mit Halbpalmetten in Rahmen mit Knospen, Eckausläufern und Palmette mittig auf dem oberen Rand, in schwarzer und roter Tinte. Marginale Zeichnungen (Federproben) auf f. 21, 36", 51", 86" 111", 116", 137; zwei marginale Zeigehände f. 170".

Einband. Moderner Ledereinband.

Erhaltungszustand. Durch Abreibung. und Feuchtigkeitseinwirkung ist der Erhaltungszustand zum größten Teil sehr schlecht. Die Schrift wurde an ausgewisehten Stellen später teilweise nachgezogen. Fehlstellen und Löcher sind zum Teil ausgebessert; Löcher f. 1; 26; 107; 138; 175; 183.

\section{Bibliographie.}

C. Korolevskij, Art. Basiliens italo-grecs et espagnols, DHGE 6 (Paris 1932) 1180-1236, bes. 1230.

G. Giovanelli, Il tipico archetipo de Grottaferrata, BollGrott n/s. 4 (1950) 17-30.98-113, bes. 19 Anm. 12; 100 Anm. 10.

S. G. Mercati, Sulle Reliquie del Monastero di Santa Maria del patire presso Rossano, in: ders., Collectanea Byzantina II (Bari 1970) 395-408 (= Archivo Storico per la Calabria e la Lucania 9 [1939] 1-14) (Teiledition).

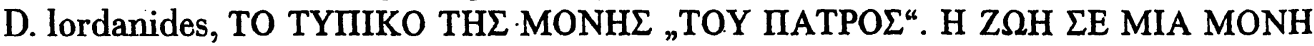




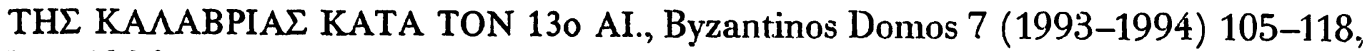
bes. $108 \mathrm{f}$.

S. Lucà, I Normanni e la ,rinascita' del sec. XII, Archivio storico per la Calabria e la Lucania 60 (1993) 1-91, bes. 11 f. u. 22.

E. Morini, Il monachesimo Italo-Greco e l'influenza di Studios, in: Ellenismo Italiota dal VII al XIl secolo. Alla Memoria di Nikos Panagiotakis (Athen 2001), 125-151, bes. 130-132 (Ankündigung einer Edition).

\section{G.B. f. 31}

Praecepta Salubria/Galen

'Spiegelblatt) Ende 18./Anfang 19. Jh./(ff. 1-8) gegen Mitte des 14. Jh.s

Papier I. 8, I' $362 \times 265 \mathrm{~mm}$

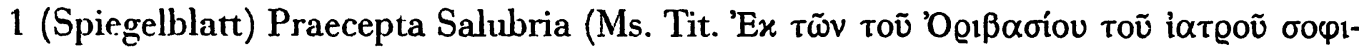

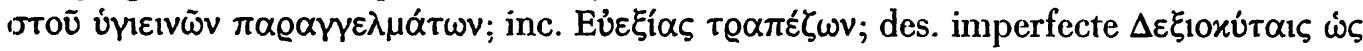

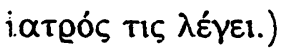

Oribasii medicinalium collectorum liber ... et fragmentum aliud e codice Mosquensi nunc primum Graece et Latine, ed. Ch. G. Gruner (Jena 1782).

L̈ber dem Text: Ex Cod. 279. Am Rand Marginalien (Konjekturvorschläge) vom Schreiber.

2 (ff. 1-8v) Galen, Linguarum seu dictionum exoletarum Hippocratis explicatio (Ms.

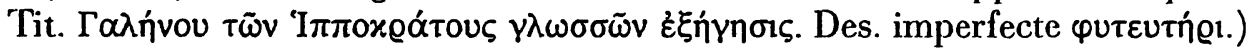
C̈ber dem Text f. 1 Notiz von Hand des 18. Jh.s: „Scriptus, ut videtur, circiter 1530. Hyq."

Textüberlieferung. Beim Text der Praecepta salubria handelt es sich um eine Abschrift des Codex Mosquensis gr. 279. Die Abschrift diente als Vorlage für die Edition von Christian Gottfried Gruner, Oribasii medicinalium collectorum liber ... et fragmentum aliud e codice Mosquensi nunc primum Graece et Latine, Jena 1782.

Die Textform von Galens Glossar ist der "classis prior" zuzurechnen und zeigt große Nähe zum Cod. Vaticanus gr. 277. Vermutlich handelt es sich um das Fragment einer Hippokrates-Handschrift.

Foliierung. Mit Bleistift 1-8.

Lagen. $1 \times 4$ : keine Kustoden vorhanden.

Beschreibstoff. Papier mit dicken Ripplinien.

I'asserzeichen. (ff. 1-8) Kreise ähnlich Briquet 3206 (Valdoule 1342) und Briquet 3205 (Dijon 1329).

Schreiber: (Spiegelblatt) 〈Chr. Fr. Matthaei〉 (auf Crund des Duktus); (f. 1-8) Hand A (vgl. Abbildung 6): Senkrechte Schrift. Ab f. $7^{*}$ Mitte wird die Schrift kleiner und der Zeilenabstand enger.

Provenienz. Bei diesem Codlex handelt es sich mit hoher Wahrscheinlichkeit um den in der C̈berlieferung von Galens Glossar immer wieder genannten, aber als verschollen geltenden Codex Mosquensis (vgl. L. Perilli, La tradizione del Glossario Ippocratico di Cáleno, in: 1 Testi Medici Creci. Tradizione e Ecdotica, Atti del Ill Convegno Internazionale. Napoli 15-18 ottobre 1997, a cura di A. Garzya e J. Jouanna [Napoli 1999] 4.31 f.). Dies ist schon dadurch nubegeleg1, daß der 'Text der Praecepta salubria auf dem Spiegelblatt von Chr. Fr. Matthaei aus Codex Mosquensis gr. 279 kopieri wurde (vgl. oben Schreiber). Hinzu kommt, daß die (valen-Lesarten des Codex Mos- 
quensis durch den Jenaer Medizinprofessor Christian Cottfried Gruner bekannt wurden (vgl. J. G. F. Franz, Erotiani, Galeni et Herodoti Glossaria in Hippocratem [Lipsiae 1780] XIX.XXII) und (größtenteils) mit denen des Jenensis übercinstimmen. Vor allem jedoch bricht der Text des Jenensis an derselben Stelle wie der des Codex Mos-

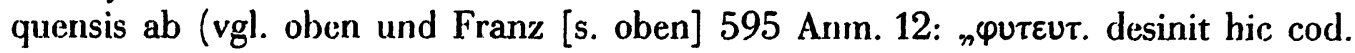
Mosq. $\left.{ }^{\circ}\right)$

\section{Linierung. keine. Lin. 39 (f. 1) - 50 (f. 8). Schriftspiegel $300 \times 240 \mathrm{~mm}$.}

Illuslralionen. f. 1 Zierleiste mit schwarz-rotem Rautenmuster, oben in der Mitte

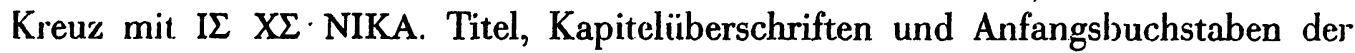
Lemmata rubriziert, Initialen in epigraphischer Auszeichnungsmajuskel.

Einband. Pappeinband, der unter Verwendung des alten Materials (aus dem 19. Jh.?' bei der Restaurierung der Handschrift 1961 angebracht wurde.

Erhaltungszustand. Die rote Tinte ist oft verblaßt bzw. ganz verschwunden. Wurmfraß. Die Handschrift war in der Mitte gefaltet; das Papier ist dort z. T. gebrochen Sie wurde 1961 restauriert.

\section{Prov. o. 25}

Griechisches Lehr- und Übungsbuch

1460-1467 Papier V, 100, III' $168 \times 110 \mathrm{~mm}$

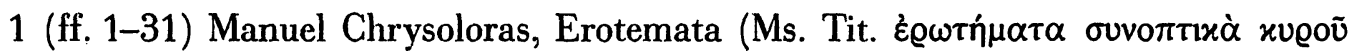

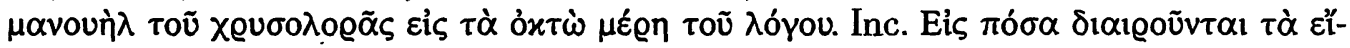

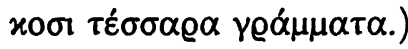

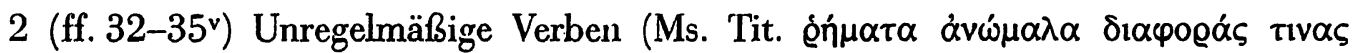

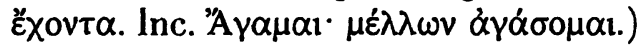

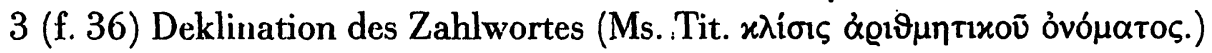

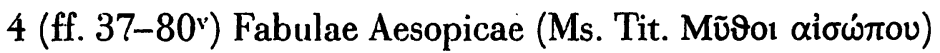

Corpus fabularum Aesopicarum, vol. 1.1 u. 1.2, ed. A. Hausrath/H. Hunger (Leipzig 21970 u. 1959).

5 (f. 83v) Martial, Epigramm 14, 183

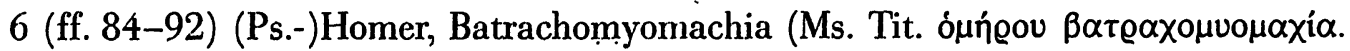
ì Tíy@itos toũ Ka@ós)

Homeri Opera Vol. V., ed. T. W. Allen (Oxford 1990), 168-183.

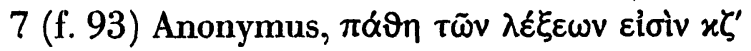

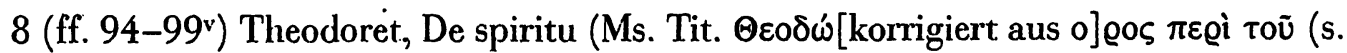

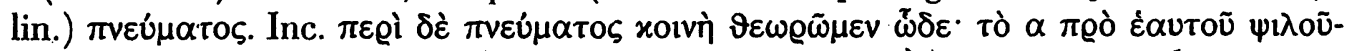

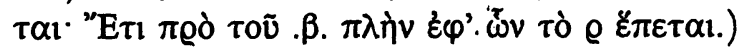

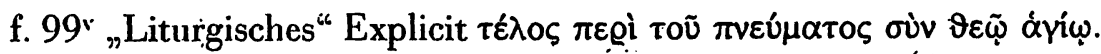

Leer: f. $31^{\mathrm{v}}, 36^{\mathrm{v}}, 81-83,92^{\mathrm{v}} ; 100^{\mathrm{v}}$.

Textüberlieferung. (ff. 1-31) Gegenüber de̊r Druckfassung (Venedig [Aldo Manutius]

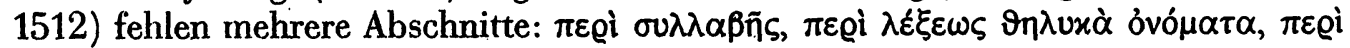

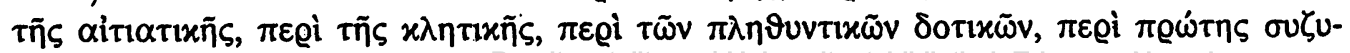




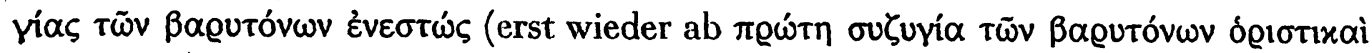

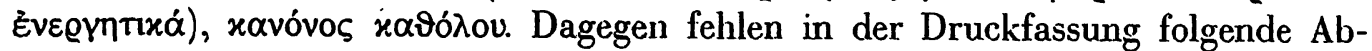

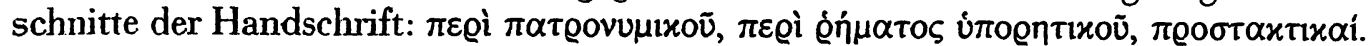

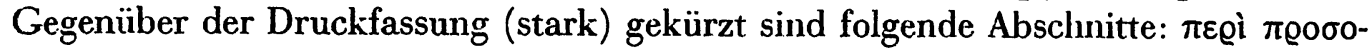

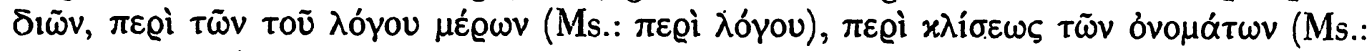

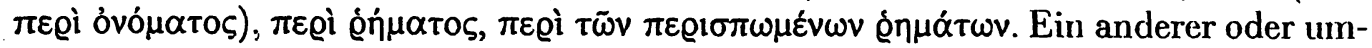

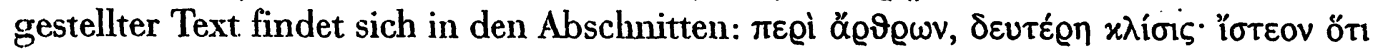

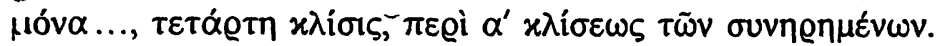

(ff. 37-80") vgl. Hausrath/Hunger (s. Bibl.)

(ff. 84-92) vgl. Allen (s. Bibl.)

Foliierung. I-IV (auf f. III auch 8); 1-100; daneben findet sich auf folgenden ff. eine weitere Zählung: f. 32 (63); 37 (73); 41 (81); 46 (91); 50` (100); 57 (111); 62 (121); 67 (131); 72 (141); 77 (151); 82 (161); 84 (165); 87 (171); 92 (181); 93 (186); 94 (187).

Lagen. $2 \times 10 ; 2 \times 12 ; 1 \times 10 ; 1 \times 14 ; 1 \times 6 ; 1 \times 10 ; 1 \times 8 ; 1 \times 10(-4)$. Keine Kustoden.

\section{Beschreibstoff. Papier.}

Wasserzeichen. (ff. 1-10) Dreiberg (kein Gegenstück gefunden), (ff. 11-54) Pfeile sehr ähnlich Piccard IX/2, 2327 (Padua 1461), (ff. 55-84) Pfeile.sehr ähnlich Piccard IX/2, 2327 (Padua 1461)/Waage ähnlich Piccard V, 383 (Landshut 1465), (ff. 85-100) Pfeile sehr ähnlich Piccard IX/2, 2327 (Padua 1461).

Schreiber. (ff. 1-92) 〈Demetrios Chalkondyles〉 (Harlfinger, auf Grund des Duktus: -vgl. Abbildung 7), vgl. Repertorium I, Nr. 105; II, Nr. 138; III, Nr. 171; (ff. 93-99") (Johannes Löffelholtz) (Harlfinger, auf Grund des Duktus; vgl. Abbildung 8), vgl. Repertorium I, Nr. 169; II, Nr. 224.

Randglossen und Textkorrekturen von 〈Demetrios Chalkondyles〉, weitere marginale griechische Notizen und Korrekturen auf ff. 9, 12v, 27, 41v, 42, 47, 61, 82v, 89, 89v von 〈Johannes Löffelholtz〉, von dessen Hand wahrscheinlich auch die lateinische Interlinearglossen stammen.

Auf Vorsatzblättern: f. II ${ }^{v}$ Abkürzungsverzeichnis der Akzente und Ligaturen, Schreibübungen, Alphabet. f. IV ${ }^{v}$ Alphabet; f. 100 Federproben.

Provenienz. Padua. Die Handschrift wurde dort von Johannes Löffelholtz 1467 erworben (vgl. den Besitzeintrag „1467 Joannes Löffelholtz de Noremberga" auf f. II ${ }^{v}$ ) und offensichtlich bearbeitet ${ }^{14}$.

Die Handschrift kursierte nach Löffelholtz' Tod in Kreisen des 'Pegnesischen Blumen' ordens in Nürnberg. Dies zeigen die weiteren Besitzvermerke: f. llla ${ }_{n}$ Sum Michaeli Satrapi Noric Anno Domini 1582. 18 Februarij $^{615}$, f. IIIIa "Sum ex Msstis. M. Christoph. Arnold. A. C. 1.6.49"16; f. 100a „Jobannes Löffelholtz" [durchgestrichen],

14 Vgl. Allgemeine Deutsche Biographie 19, 1884; 90-99.

1.5 Diese Person ist nicht identifizierbar; evtl. handelt es sich um Michael Vitzthum oder dessen Vater. vgl. C. A. Will, Nürnlsergisches Celehrten-Lexikon IV (Altdorf 1758) 95. Vitzthum stanitnte aus Lauf, inmatrikulierte sich am 16. 5. 1603 in Altdorf. Dort hielt or an 18. 3. 1609 eine gricchi-

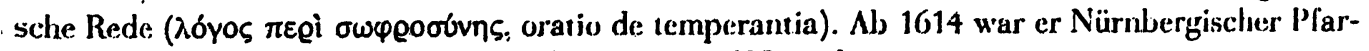
rer in Buschendorf, ab 1620 in Tennenlohe. wo er 1622 starb.

$10 \mathrm{Vgl}$. Algemeine Deutsche Biographie 1, 1875, 584; Deutsche biographisclıe Esız'klopädie 1, 1995. 185. 
"Johannes Geuder de Norimberga" ${ }^{\prime 17}$. Wie sie dann in den Besitz Griesbachs (vgl. den Besitzvermerk auf f. Ia „Bibliothecae Academicae D. D. Jo. Jac. Griesbach Theol. D. \& P. P. O. Jenae, Cal. Octobr. MDCCLXXVIII.") kam, ist nicht mehr zu rekonstruieren.

Linierung. keine. Lin.: 18. Schriftspiegel: $115 \times 72 \mathrm{~mm}$.

Illustrationen. Titel und Initialen in braunroter Tinte.

Einband. Lederüberzogener Pappband des 18. Jh. mit Goldprägung auf dem Rücken und Aufdruck: "Chrysoloras | Aesopus | Batracho-|myom. | Mss. Gr."

Erhaltungszustand. Schäden an den Blatträndern (stark abgegriffen) - teils ausgebessert; Wasserschäden. Korrekturen; Ausbesserung ausgewischter Textstellen; geringer Wurmfraß.

Bibliographie.

A. Ludwich, Die homerische Batrachomachia des Karers Pigres nebst Scholien und Paraphrase (Leipzig 1896) 47 Nr. 43.

Corpus fabularum Aesopicarum, Vol. I, ed. A. Hausrath (2. Aufl. H. Hunger), fasc. 1 (Leipzig 1940/1970) XII.

Homeri Opera Vol. V., ed. D. B. Monro/T. W. Allen (Oxford 1963), 165 Nr. 21.

INDEX

\section{Personen}

Arnold, C.

Barth, K. v.

Bose, J. A.

Chalkondyles, $\mathrm{D}$.

Cornarius, J.

Geuder, J.

Goethe, W. M. Frhr. v.

Griesbach, J. J.

Gruner, C. G.

Johannes

Werke

Epiphanius von Salamis

Panarion

De fide

Ancoratus

De mensuribus et ponderibus

(Ps.-)Epiphanius von Salamis

Anacephalaeosis.

Fabulae Aesopicae

Galenus

Hippocrates-Lexikon

(Ps.-)Homer
Prov. 0. 25

Bos. f. 1

Bos. f. 1; Bos.

q. 7

Prov. o. 25

Bos. f. 1

Prov. o. 25

G.B. q. $6^{\mathrm{a}}$

Prov. o. 25

G.B. f. 31

Bos. q. 7

Bos. f. 1

Bos. f. 1

Bos. f. 1

Bos.f. 1

Bos. f. 1

Anm. 6;

Prơvv. o. 25

G.B. f. 31
Lang, J.

Löffelholtz, J.

Matthaei, C. F.

Melanchthon, Ph.

Oporinus, J.

Satrapa, M.

Schnuck, J.

Schmuck, W.

Trithemius, $\mathrm{J}$.
Bos. f. 1

Prov. 0. 25

G.B. f. 31

Bos. f. 1

Bos. f. 1

Prov. o. 25

Bos. q. 7

Bos. q. ?

Bos. f. 1
(Ps.-?)Johannes Chrysostomus

De duobus testamentis Bos. f. 1

Sermones II prophylatici Bos. f. 1

Manuel Chrysoloras

Eroternata

Prov. o. 25

Martial

Epigramm 14,183

Nicephorus von Konstantinopel

Chronographicon synton on Bos. f. 1

Praecepta salubria

Sophoclés

17 Vgl. Deutsche biographische Enzyklopädie 3, 1996, 670. 


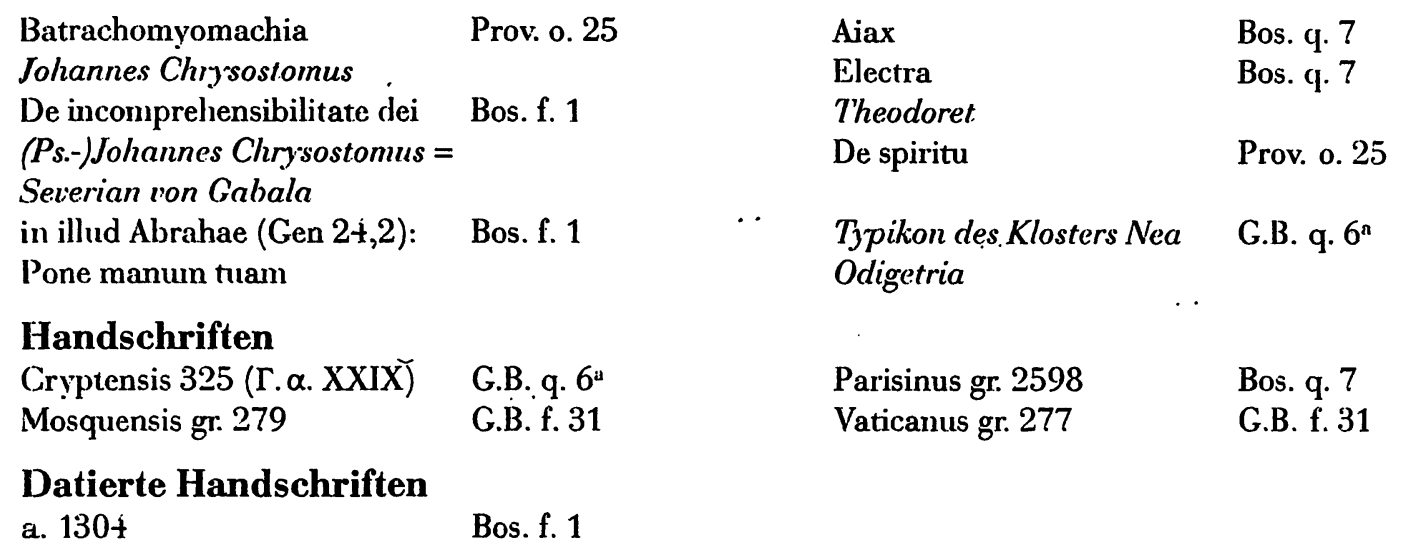

